\title{
Clinicopathological Features and Prognosis of Gastric Cancer in Young Patients
}

\author{
Ki Bum Park, M.D., Kyong Hwa Jun, M.D., Ph.D. \\ Department of Surgery, St. Vincent's Hospital, The Catholic University of Korea, Seoul, Korea
}

\begin{abstract}
Gastric cancer mainly occurs in middle-aged and older people after their 50s, and the incidence of gastric cancer in younger people is rare. The frequency of gastric cancer that occurred before 40 years of age is $6-8 \%$ of all gastric cancer patients, and most of them are over 35 years of age. The prognosis of gastric cancer in young patients is believed to be poor because of more aggressive tumor behaviors and delayed diagnosis, however, there is controversy.
\end{abstract}

Keywords: Gastric cancer, Prognosis, Diagnosis, Young patient

\author{
Received November 3, 2020 \\ Revised November 18, 2020 \\ Accepted December 7, 2020 \\ Corresponding author \\ Kyong Hwa Jun \\ Department of Surgery, St. Vincent's \\ Hospital, College of Medicine, The \\ Catholic University of Korea, 93 \\ Jungbu-daero, Paldal-gu, Suwon \\ 16247 , Korea \\ Tel: +82-31-881-8636 \\ Fax: +82-31-547-5347 \\ E-mail: dkkwkh@catholic.ac.kr \\ ORCID: \\ https://orcid.org/0000-0003-3909-5230
}

Copyright $(2020$ The Journal of Minimally Invasive Surgery. All rights reserved. This is an Open Access article distributed under the terms of the Creative Commons Attribution Non-Commercial License (http://
creativecommons.org/licenses/by-nc/4.0/) which permits unrestricted non-commercial use, distribution, and reproduction in any medium, provided the original work is properly cited.

Males have a higher incidence of gastric cancer than females with a ratio approximately $2: 1$, but gastric cancer in young patients exhibited a female predominance. ${ }^{1}$ Young gastric cancer patients are thought to exhibit specific clinicopathological characteristics and more aggressive tumor biology than older patients. These characteristics include an increased proportion lesions in the upper third of the stomach, mixed and diffuse types (based on Lauren classification), significantly more signet ring cell types and poorly differentiated histologic types, and Borrmann type $\mathrm{IV}^{2}$ However, delay in diagnosis is the main reason of more advanced stages because young gastric cancer patients usually lack early symptoms and the health screening system for gastric cancer rarely considers these young populations.

Lymph node metastasis in gastric cancer represents the strongest clinicopathological factor regarding survival. Recently, several models or scoring systems to predict the lymph node metastasis in gastric cancer has been developed. ${ }^{3}$ Most models were consistent with several significant clinical factors but different in the clinical usefulness of results and metastatic lymph node location. ${ }^{3}$ Clinicopathological factors known to be associated with lymph node metastasis is depth of invasion, size, histological type, morphological classification, venous, and lymphatic invasion. ${ }^{4}$ Histologically, most young gastric cancer patients had undifferentiated histologic types and advanced lymph node metastasis. Because undifferentiated gastric cancer infiltrates more prominently in a vertical direction and frequently shows lymph node involvement, then Borrmann types III and IV would be more common in young gastric cancer patients, thus conferring a high risk of lymph node metastasis. ${ }^{4}$ In the aspect of these findings, lymph node dissection might significantly be important in 
the surgical treatment of young gastric cancer patients. In this issue of J Minim Invasive Surg, Kim et al. ${ }^{5}$ reports younger group was an independent risk factor for nodal metastasis and an independent risk factor for clinical N-stage underestimation. Authors evaluated clinical staging using the preoperative computed tomography (CT) and endoscopic ultrasonography (EUS) from the patient medical records and clinical stage was considered conservatively; higher stage of the two exams were selected. Both the younger group and the older group had similar operations, and the majority of patients in both groups underwent D2 lymph node dissection (61.1\% vs $62.5 \%)$.

The prognosis of young gastric cancer patients has been controversial for decades. It is thought that young gastric cancer patients exhibit a poor prognosis and lower overall survival rate compared with older patients due to more aggressive tumor biology. However, several recent studies demonstrated the prognosis in young gastric cancer patients was equal to or better than that of the older gastric cancer patients. ${ }^{1-4}$ Authors showed the overall 5 -year survival for the older group was significantly lower than that for the younger group. ${ }^{5}$ And there was no significant difference in 5-year cancer-specific survival for both groups. There might be several reasons that have driven the authors' results. ${ }^{4}$ First, delay in diagnosis was one of the main causes of more advanced stages in young gastric cancer patients, resulting in worse prognosis. However, the overall survival of young gastric cancer patients was improved because advanced diagnostic techniques improved the early diagnostic rate of young patients. Second, physicians paid closer attention to young patients and try more aggressive treatment options for young gastric patients with advanced stages.

\section{ORCID}

Ki Bum Park, https://orcid.org/0000-0001-6035-6584

Kyong Hwa Jun, https://orcid.org/0000-0003-3909-5230

\section{AUTHORS' CONTRIBUTIONS}

Conceptualization: Kyong Hwa Jun. Formal analysis: Kyong Hwa Jun. Methodology: Ki Bum Park and Kyong Hwa Jun. Writing-original draft: Ki Bum Park and Kyong Hwa Jun. Writingreview and editing: Kyong Hwa Jun.

\section{CONFLICT OF INTEREST}

None.

\section{FUNDING}

None.

\section{ACKNOWLEDGMENTS}

None.

\section{REFERENCES}

1) Jun $\mathrm{KH}$, Im MG, Won YS, et al. A clinicopathological analysis of gastric cancer in young patients. J Korean Surg Soc 2005;69:217-223.

2) Takatsu $Y$, Hiki N, Nunobe $S$, et al. Clinicopathological features of gastric cancer in young patients. Gastric Cancer 2016;19:472-478.

3) Kim $\mathrm{SM}$, Lee $\mathrm{H}$, Min $\mathrm{BH}$, et al. A prediction model for lymph node metastasis in early-stage gastric cancer: Toward tailored lymphadenectomy. J Surg Oncol 2019;120:670-675.

4) Wang Z, Xu J, Shi Z, et al. Clinicopathologic characteristics and prognostic of gastric cancer in young patients. Scand J Gastroenterol 2016; 51:1043-1049.

5) Kim W, Youn S, Won Y, et al. Clinicopathologic characteristics of young gastric cancer patients: Diagnostic staging accuracy and survival. J Minim Invasive Surg 2020;23:163-171. 